ITEP-TH-7/99

\title{
Solitons on Branes
}

\author{
G. W. Semenofft and K. Zarembot \\ The Niels Bohr Institute \\ Blegdamsvej 17 \\ DK-2100 Copenhagen 0 \\ Denmark
}

\begin{abstract}
We examine the possibility that gauge field configurations on stacks of parallel Dp-branes support topological solitons. We give an exhaustive list of possible soliton charges for $p \leq 6$. We also discuss how configurations carrying the soliton charges can be constructed from intersecting branes.
\end{abstract}

\section{Introduction}

One of the most intriguing features of D-branes is that they provide the possibility of embedding super-symmetric gauge theories in various dimensions into string theory [1] (see [2] for a review). In many cases, these field theories possess soliton states which are associated with topologically non-trivial configurations of the fields. The fact that the gauge fields on D-branes can form such topologically non-trivial configurations appears to be important in

${ }^{*}$ Research supported by NSERC of Canada and the Niels Bohr Fund of Denmark and NATO Grant CRG 970561. Permanent address: Department of Physics and Astronomy, University of British Columbia, 6224 Agricultural Road, Vancouver, British Columbia, Canada V6T 1Z1. E-mail: semenoff@alf.nbi.dk

${ }^{\dagger}$ Research supported by a NATO Science Fellowship, NSERC of Canada, INTAS grant 96-0524, RFFI grant 98-01-00327. Permanent address: Department of Physics and Astronomy, University of British Columbia, 6224 Agricultural Road, Vancouver, British Columbia, Canada V6T $1 Z 1$ and Institute for Theoretical and Experimental Physics, B. Cheremushkinskaya 25, 117259, Moscow, Russia. E-mail: zarembo@alf.nbi.dk 
the interpretation of D-brane charges [3, 4, 5, 6, 7] and potentially could lead to a deeper understanding of the origin of the D-branes themselves $[8,4,19,10,11,12$.

The topological charge on the world-volume of a D-brane couples to the space-time Ramond-Ramond fields of type II superstring theory via Chern-Simons terms in the worldvolume action [13, 14, 15]. This allows an alternative interpretation of the topological charge as the RR charge due to the presence of lower-dimensional branes which are either suspended between the higher dimensional branes [16] or embedded in the world-volume of the higher dimensional branes [13]. An example is the description of BPS magnetic monopoles in $d=4, \mathcal{N}=4$ super-Yang-Mills theory which can be viewed either as conventional BPS monopoles of the Coulomb branch of the gauge theory or as D-strings stretched between parallel D3-branes 16, 17. Also, instantons in that theory can be described alternatively as either conventional Yang-Mills instantons or (the T-dual of) D0-branes immersed in the world-volume of D4-branes [13, 18, 19, 20, 21]. Our aim in this Paper is to give a complete topological classification of world-volume defects and their string interpretation for all stable BPS Dp-brane states in type IIA and IIB string theories, with $p \leq 6$ and which preserve half of the ten-dimensional $\mathcal{N}=2$ super-symmetry.

The most general configurations of this type contain $m$ parallel Dp-branes each carrying RR charges $N_{1}, \ldots, N_{m}$. In the limit when their positions coincide, the internal symmetry group of such a configuration is $G=U(N)$ [1], where $N=\sum N_{i}$ is the total RR charge. When the branes are separated to form parallel stacks of $N_{1}, N_{2}, \ldots$ coinciding branes, this symmetry is spontaneously broken to

$$
H=U\left(N_{1}\right) \times \ldots \times U\left(N_{m}\right)
$$

This situation generically allows for the existence of topological defects 22]. The soliton states arise because the space of classical vacua is a manifold with non-trivial topology. The vacua, which are field configurations of the supersymmetric gauge theory with zero potential energy, are flat connection gauge fields residing in the unbroken subgroup of the gauge group and values of scalar fields which minimise the potential. Finiteness of the energy imposes boundary conditions on any legitimate field configuration, requiring it to take vacuum values at infinity. The possible boundary conditions are classified by topologically distinct mappings from the sphere at infinity to the space of vacua. This leads to the existence of topologically distinct sectors in the space of all field configurations. The charges which distinguish these sectors take values in the $\pi_{p-1}$ homotopy group of the vacuum manifold.

The instanton charges are related to topologically non-trivial pure gauge fields of the unbroken subgroup and are classified by

$$
\pi_{p-1}\left(U\left(N_{1}\right) \times \ldots \times U\left(N_{m}\right)\right)
$$

Another type of topological defect, called a topological soliton, is classified by mappings from the sphere at infinity to the space of equilibrium values of internal scalar fields. If the positions of branes are held fixed, the classical vacua are parametrised by the coset space $U(N) / U\left(N_{1}\right) \times \ldots \times U\left(N_{m}\right)$ and the soliton states are classified by the homotopy groups

$$
\pi_{p-1}\left(U(N) / U\left(N_{1}\right) \times \ldots \times U\left(N_{m}\right)\right)
$$


We remind the reader that topological arguments alone do not guarantee the existence of stable solutions of the classical equations of motion in a given topological sector.

The homotopy groups of a coset space $G / H$ occur in exact sequences of the fibration $G \rightarrow G / H:$

$$
\ldots \longrightarrow \pi_{p-1}(G) \stackrel{f_{1}}{\longrightarrow} \pi_{p-1}(G / H) \stackrel{f_{2}}{\longrightarrow} \pi_{p-2}(H) \longrightarrow \ldots
$$

In all of the cases which we shall consider, the exactness of this sequence unambiguously fixes the necessary homotopy groups. Also, certain information about the nature of the resulting topological solitons can be obtained from the structure of the homomorphisms $f_{1}$ and $f_{2}$.

If $f_{1}$ is an isomorphism, the corresponding topological defects are instantons of the broken gauge group [23]. Indeed, the homomorphism $f_{1}$ is induced by the projection map $G \rightarrow G / H$. An explicit realization of the projection map is

$$
U \mapsto U^{-1} \phi^{i} U \quad
$$

where $\phi^{i}$ is a set of diagonal $N \times N$ matrices. Interpreting the eigenvalues of $\phi^{i}, i=1 \ldots 9-p$ as transverse coordinates of $N$ D-branes and denoting by $\Phi^{i}$ corresponding scalar fields in the world-volume theory, we find that a field configuration going to the pure gauge at infinity:

$$
\Phi^{i} \longrightarrow U^{-1} \phi^{i} U, \quad A \longrightarrow U^{-1} d U
$$

carries a topological charge taking values in $\pi_{p-1}(G / H)$. The homotopy class of this configuration coincides with the homotopy class of the gauge transformation $U$ at infinity which takes values in $\pi_{p-1}(G)$.

For $N$ sufficiently large, the homotopy groups responsible for instantons are periodic in $p$ [24]:

$$
\pi_{1}(U(N))=\pi_{3}(U(N))=\pi_{5}(U(N))=Z .
$$

Thus, topological arguments suggest that there are instantons on even-dimensional branes. There are following exceptions for low $N$ :

$$
\pi_{k}(U(1))=0(k>1), \quad \pi_{4}(U(2))=\pi_{5}(U(2))=Z_{2} .
$$

The exceptional case of $N=2, p=5$ is discussed in Sec. 6 .

On the string side, instantons are described by D0-branes embedded inside Dp-branes [13, 18, 19, 25, 20, 21]. As was mentioned in [4], the Bott periodicity (1.2) of the homotopy groups $\pi_{n}(U(N))$ appears to be encoded in the string description, since 0-branes exist in type IIA string theory and can only couple to even-dimensional branes.

If $f_{2}$ is an isomorphism, so that

$$
\pi_{p-1}(G / H)=\pi_{p-2}(H)
$$

or, more generally, if $f_{2}$ is an onto mapping, so that

$$
\pi_{p-1}(G / H)=\pi_{p-2}(H) / \pi_{p-2}(G)
$$

the corresponding defects are generalised monopoles, as explained in Sec. 2, and the generic case is described by open D1-branes stretched between adjacent higher-dimensional branes. 
Again, the D-brane picture matches the topological consideration, since, on the string side, we expect to find monopoles on odd-dimensional branes of type IIB theory. On the other hand, the stable homotopy groups responsible for monopoles are

$$
\pi_{p-1}\left(U(N) / U\left(N_{1}\right) \times \ldots \times U\left(N_{m}\right)\right)=\left\{\begin{array}{ll}
Z^{m-1}, & p \text { odd } \\
0, & p \text { even }
\end{array} .\right.
$$

For completeness, we sketch the calculation of these groups in Appendix A.

\section{Monopoles on D-branes}

The potential of the super-Yang-Mill's theory has the form

$$
V \propto \operatorname{Tr}\left(\sum_{i}\left(D \Phi^{i}\right)^{2}-\sum_{i<j}\left[\Phi^{i}, \Phi^{j}\right]^{2}\right)
$$

and is minimised by classical fields $\Phi^{i} i=1, \ldots, 9-p$ which commute with each other and are therefore simultaneously diagonalisable. Their distinct eigenvalues, $\phi_{a}^{i}, a=1, \ldots, m$, each with degeneracy $N_{1}, . ., N_{m}$ are the positions of the $m$ stacks of parallel $D p$-branes. In a field configuration with a topological defect, the scalar fields must minimise the potential at infinite distances from the location of the defect and deviate from the minimum inside the core. Even though the eigenvalues of $\Phi^{i}$ must therefore approach constants at infinity, generally, the scalar fields themselves approach non-diagonal matrices $\Phi^{i}=U^{-1} \phi^{i} U$. Furthermore, to minimise the classical energy, these matrices must be covariant constants. The gauge fields therefore approach pure gauges $A \rightarrow U^{-1} d U$.

Generally, $U$ is not defined globally on the sphere at infinity, $S^{p-1}$, but must have a singularity at some point on $S^{p-1}$. In this case, the singular gauge transformation which passes to the unitary gauge by removing $U$ and leaving $\Phi^{i}$ diagonal creates a Dirac string. In this gauge, both the Dirac string and the gauge field at infinity carry magnetic fluxes in the unbroken subgroup $H$. This is a result of the fact that, from the definition of the boundary homomorphism $f_{2}$ [24], the singularity of $U$ resides in the unbroken subgroup $H$. This defines a mapping $V$ from the sphere $S^{p-2}$ which links the intersection of the Dirac string with $S^{p-1}$ to $H$. If the homotopy class of this mapping is represented by an integral over $S^{p-2}$ of the $(p-2)$-form $\operatorname{tr} \omega^{p-2}$, where $\omega=V^{-1} d V$, then it is equal to the magnetic

flux through the sphere at infinity, $\int_{S^{p-1}} \operatorname{tr} F^{(p-1) / 2}$, where $F$ is the field strength in the unbroken subgroup and the gauge field approaches $\omega$ on $S^{p-2}$. The structure of the mapping $f_{2}$ between homotopy groups is such that, when it is an isomorphism, the magnetic flux in the unitary gauge is equal to the topological charge of the defect. The corresponding topological defects are generalised magnetic monopoles. If $f_{2}$ is not an isomorphism, the topological charge is equal to the magnetic flux modulo an instanton number of the gauge field $A$.

These generalised monopoles are described by D-strings stretched between stacks of Dpbranes, where $p$ is odd. To show that such configurations of branes are possible, we have to slightly generalise the rules for D-strings to end on Dp-branes [26] to allow for coincident higher-dimensional branes. 
The derivation follows [16] and is based on the fact that RR two-form $C$ couples to odd-dimensional branes via world-volume Chern-Simons term [13]:

$$
I=\int_{D 1} C \wedge \operatorname{tr} \mathbf{1}+\frac{1}{2 \pi} \int_{D 3} C \wedge \operatorname{tr} F+\frac{1}{8 \pi^{2}} \int_{D 5} C \wedge \operatorname{tr} F \wedge F+\ldots,
$$

where $F$ is the field strength of the internal gauge field. Consequently, the total two-form $\mathrm{RR}$ charge density is equal to

$$
J^{i 0}(y)=k \int_{D 1} d x^{i} \delta(y-x)+\frac{1}{2 \pi} \int_{D 3} d x^{i} \wedge \operatorname{tr} F \delta(y-x)+\frac{1}{8 \pi^{2}} \int_{D 5} d x^{i} \wedge \operatorname{tr} F \wedge F \delta(y-x)+\ldots,
$$

where $k$ is the $\mathrm{RR}$ charge of the $\mathrm{D}$-string. If the D-string has an end point on a higher dimensional D-brane, the charge conservation condition,

$$
\partial_{i} J^{i 0}=0,
$$

can only be satisfied if the field strength $F$ has a singularity at the point where the D-string terminates. Integrating over an eight-sphere surrounding the end point of the D-string, we get:

$$
k= \begin{cases}-\frac{1}{2 \pi} \int \operatorname{tr} F, & \text { if the D-string terminates on a D3-brane } \\ -\frac{1}{8 \pi^{2}} \int \operatorname{tr} F \wedge F, & \text { if the D-string terminates on a D5-brane } \\ \cdots & \end{cases}
$$

where the integral is over the sphere surrounding the end-point of the D-string on the Dpbrane. The right hand side is the monopole charge, so, the end point of a D-string looks like a magnetic monopole.

The above consideration concerns D-strings ending on one stack of Dp-branes. The Dstring stretched between two stacks of $N_{1}$ and $N_{2}$ coincident branes describes a monopole of $U\left(N_{1}\right)$, which corresponds to one end of the string, and an anti-monopole of $U\left(N_{2}\right)$, which corresponds to the other end. The monopole arising due to symmetry breaking $U\left(N_{1}+N_{2}\right) \rightarrow$ $U\left(N_{1}\right) \times U\left(N_{2}\right)$ has precisely the same quantum numbers.

In the subsequent sections we classify all possible defects, both monopoles and instantons, on Dp-branes with $p \leq 6$.

\section{2-branes}

The only possible defects on D2-branes are instantons classified by $\pi_{1}(U(N))=Z$. Obviously, they are associated with $U(1)$ factor in $U(N)$. Relevant degrees of freedom are described at low energies by free super-symmetric Abelian gauge theory. The localised classical field configurations carrying the instanton charge are unstable in the free theory. This instability in the D-brane picture is reflected in the fact that the fundamental string has a tachyonic mode for a 0-brane embedded in the world-volume of a 2-brane [27].

It is worth mentioning that D0-branes embedded in Dp-branes can also be associated with delocalized magnetic flux [28, 29, 30, 31, 32] and a 2-brane carrying a 0-brane charge can be described in this way [28]. The configurations of this type either require the finiteness of the D-brane volume or carry an infinite RR charge and do not fall into the class of topological defects that we consider. 


\section{3-branes}

The only non-trivial $\pi_{2}$ group is $\pi_{2}(G / H)=\pi_{1}(H) / \pi_{1}(G)=Z^{m-1}$, which is responsible for magnetic charges in $\mathcal{N}=4, d=4$ Yang-Mills theory. Different states carrying these charges were studied from the string-theory point of view in great detail [17, 33, 34, 35, 36, 37, 38].

\section{4-branes}

The D4-branes possess internal instanton charges associated with $\pi_{3}(U(N))=Z$. It worth noting that we regard instantons as static field configurations with finite energy, rather than Euclidean solutions with finite action in one dimension lower. These two types of topological defects are related by T-duality in the time direction which transforms bound states of D0 and Dp branes into bound states of D-instantons and $\mathrm{D}(\mathrm{p}-1)$ branes, configurations recently discussed in the context of AdS/CFT correspondence [39, 40, 41, 42, 43, 44, 45, 46, 32.

If there are coincident branes (some $N_{i} \geq 2$ ), the instantons can always be colour-rotated to the unbroken subgroup and there are stable classical solutions with any instanton charge. The homotopy group $\pi_{3}(G / H)$ is trivial in this case. The reasoning is the same as in Appendix A. If all the branes are separated $\left(N_{i}=1\right)$ we have the short exact sequence

$$
\begin{gathered}
\pi_{p-1}\left(U(1)^{N}\right) \longrightarrow \pi_{p-1}(U(N)) \stackrel{f_{1}}{\longrightarrow} \pi_{p-1}\left(U(N) / U(1)^{N}\right) \\
\quad \| \\
0
\end{gathered}
$$

Thus, $\pi_{3}\left(U(N) / U(1)^{N}\right)=\pi_{3}(U(N))$ and corresponding topological defects are instantons of the broken group.

Instantons in the Higgs model, as classical solutions of the field equations, are unstable and tend to shrink to a point. The size of an instanton and other instanton moduli are described by vacuum expectation values of fields on the 0-brane world line coming from

fundamental strings stretched between the 0 - and 4-branes [19, 20, 46]. The reason for the instability of an instanton on separated branes is the generation of masses for moduli fields (except for the instanton coordinates) proportional to the separation between the 0- and 4-branes [20, 46]. The existence of these masses is what forces the instanton to shrink to a point.

\section{5-branes}

It is necessary to consider the cases $N=2$ and $N>2$ separately. 


\section{1 $N>2$}

If $N>2$, the homotopy groups $\pi_{4}(G / H)$ can be determined from the long exact sequence

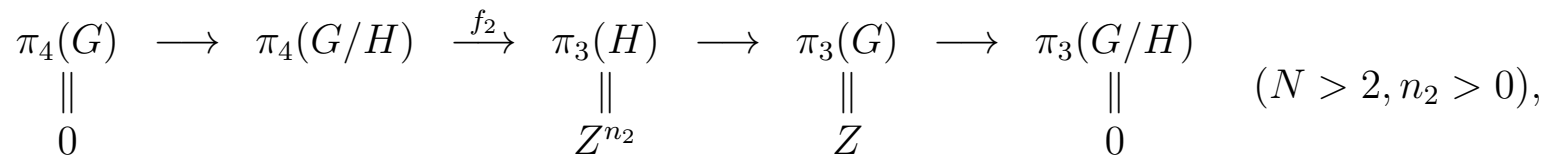

where $n_{2}$ is the number of branes with $\mathrm{RR}$ charge $N_{i} \geq 2$. We assume that $n_{2}>0$, otherwise $\pi_{4}(G / H)=0$, as follows from the same exact sequence. We find that

$$
\pi_{4}(G / H)=\pi_{3}(H) / \pi_{3}(G)=Z^{n_{2}-1} .
$$

Therefore, the homotopy groups are responsible for monopoles. The states carrying monopole charges are described by D-strings stretched between adjacent stacks of coincident branes.

\section{2 $N=2$}

The homotopy groups $\pi_{4}(U(2))=\pi_{4}\left(U(2) / U(1)^{2}\right)=Z_{2}$ (the second equality follows from the short exact sequence (5.1)) allow for $Z_{2}$ instantons on 5-branes\$. In the field theory language, the non-trivial element of $\pi_{4}(U(2))$ can be represented as an instanton-anti-instanton pair rotated through $2 \pi$ and then annihilated [48].

As far as $\pi_{4}$ is considered, we can make no difference between $U(2)$ and $S U(2)=S^{3}$. The topological classification of maps from $S^{4}$ to $S^{3}$ is given by Freudenthal theorem 49. The "easy part" of the Freudenthal theorem states that the suspension map

$$
\Sigma: \pi_{3}\left(S^{2}\right) \longrightarrow \pi_{4}\left(S^{3}\right)
$$

is an epimorphism. It follows from the "difficult part " of the Freudenthal theorem that the kernel of this epimorphism is the mapping with the instanton number 2, which infers $\pi_{4}\left(S^{3}\right)=Z_{2}$. The suspension of the mapping with the instanton number 1 represents the only non-trivial element of $\pi_{4}\left(S^{3}\right)$.

To construct the non-trivial map from $S^{4}$ to $S^{3}$ we can use the isomorphism $\pi_{3}\left(S^{3}\right)=$ $\pi_{3}\left(S^{2}\right)$. If $U(x)$ represents the canonical generator of $\pi_{3}(S U(2))$, then $\alpha: x \mapsto U^{-1} \sigma^{3} U$ defines the mapping from $S^{3}$ to $S^{2}$, the suspension of which, $\Sigma \alpha$, is the unique non-trivial element of $\pi_{4}\left(S^{3}\right)$. Explicitly, the suspension of $\alpha$ can be realized as a mapping $\Sigma \alpha:(x, \tau) \mapsto$ $U^{-1} \mathrm{e}^{2 \pi i \sigma^{3} \tau} U$.

If $\tau$ is thought of as one of the coordinates on the 5-brane, the gauge transformation at infinity which is equal to unity for $\tau<0$ and for $\tau>1$ and to $V=U^{-1} \mathrm{e}^{2 \pi i \sigma^{3} \tau} U$ for $0<\tau<1$ defines non-trivial element of the homotopy group $\pi_{4}(U(2))$. The field configuration which goes to the pure gauge

$$
\Phi \longrightarrow V^{-1} \phi V, \quad A \longrightarrow V^{-1} d V
$$

at infinity will carry $Z_{2}$ charge. Here $\phi=L \sigma^{3}$, where $L$ is the distance between branes. For a given $\tau$ the fields in this configuration are that of an instanton-anti-instanton pair

\footnotetext{
${ }_{\ddagger}^{\ddagger}$ Discrete theta-angle in 5-dimensional field theory on a 4-brane associated with $\pi_{4}(U(2))$ was discussed in 47 .
} 


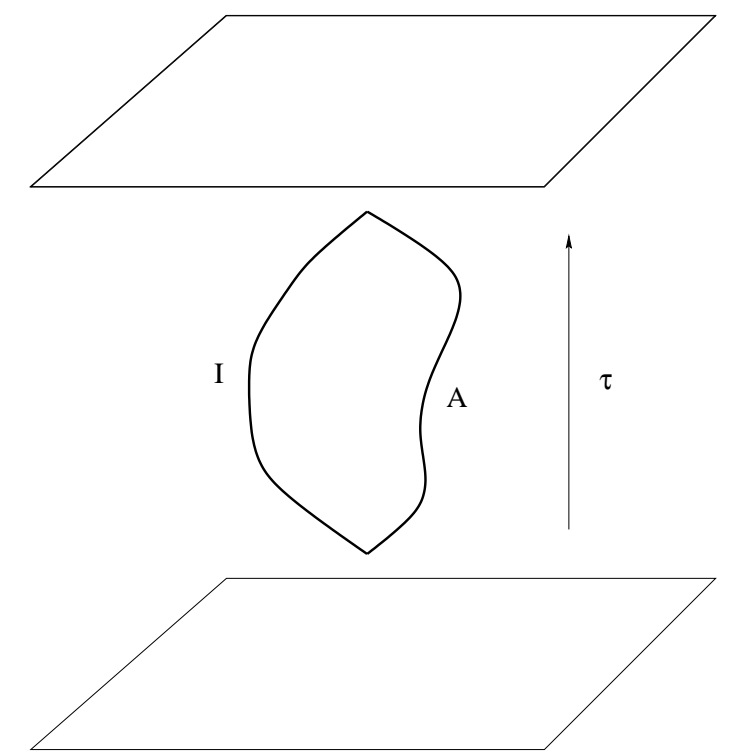

Figure 1: Instanton-anti-instanton pair in the 5-brane volume.

with the instanton rotated through the angle $2 \pi \tau$, since the fields of the rotated instanton define the pure gauge at infinity $\mathrm{e}^{2 \pi i \sigma^{3} \tau} U$, while the anti-instanton corresponds to the pure gauge $U^{-1}$. Thus, $Z_{2}$ instanton on two 5-branes can be constructed as follows. At some point inside 5-brane volume the instanton-anti-instanton pair is created, then instanton and anti-instanton are separated and the instanton is adiabatically rotated through the angle $2 \pi$, after which the pair is annihilated (fig. 1).

This interpretation readily suggests a brane picture of the $Z_{2}$ instanton. The trajectory of an instanton corresponds to the D1 brane, as can be established using T-duality. The gauge orientation of the instanton is described by a vacuum expectation value of one of the massless modes of the 1-5 fundamental strings. Thus, we conclude that the $Z_{2}$ instanton is a closed D1 brane inside two coincident D5 branes (or between two separated D5 branes) with a non-trivial configuration of low-energy modes of the fundamental string stretched between the D1 and D5 branes. It is these modes of the fundamental string that are responsible for the topological stability of the $Z_{2}$ instanton. The topological stability does not imply that there is a non-singular field configuration with minimal energy in the sector with non-trivial $Z_{2}$ charge. The D-string loop, most probably, will tend to shrink to a point, although it will not be able to annihilate.

\section{6-branes}

As follows from (1.2), (5.1), $\pi_{5}(U(N))=Z=\pi_{5}\left(U(N) / U(1)^{N}\right)$, except for $N=2$ when $\pi_{5}(U(2))=Z_{2}=\pi_{5}\left(U(2) / U(1)^{2}\right)$. These homotopy groups are responsible for instantons ( $Z_{2}$-instantons in $N=2$ case) on D6 branes. Scaling arguments show that localised instantons are unstable [25]. On the string side, this is reflected in the fact that D0 branes always repel from D6 branes [27]. Again, as in the case of instantons on D2 branes, it is possible to 
construct delocalized stable solutions of low-energy equations of motion [25].

Considering the homotopy groups $\pi_{5}(G / H)$ we have to distinguish two cases: $n_{3}>0$ and $n_{3}=0$. Here $n_{3}$ denotes a number of D6 branes with RR charge $N_{i} \geq 3$.

\section{$7.1 \quad n_{3}>0$}

Consider the exact sequence

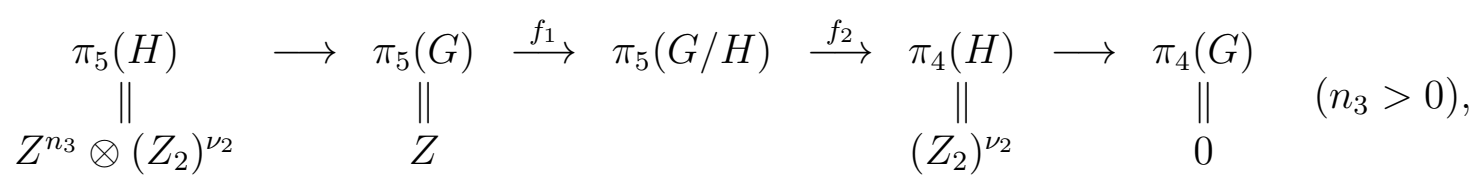

where $\nu_{2}$ is the number of branes with $\mathrm{RR}$ charge $N_{i}=2$. Since the generator of the homotopy group $\pi_{5}(U(N))$ can be represented by a $U(N)$ matrix of the form

$$
U(x)=\left(\begin{array}{cc}
\Omega(x) & 0 \\
0 & \mathbf{1}
\end{array}\right),
$$

where $\Omega$ is a $3 \times 3$ matrix, the first homomorphism in the chain (7.1) covers the entire $\pi_{5}(G)$ group. Hence, $f_{1}$ is a trivial mapping: $\operatorname{Im} f_{1}=0$, and the boundary homomorphism $f_{2}$ is an isomorphism in this case:

$$
\pi_{5}(G / H)=\pi_{4}(H)=\left(Z_{2}\right)^{\nu_{2}} .
$$

Thus, there are $\nu_{2}$ species of $Z_{2}$ monopoles on such configurations of D6 branes.

We should impose unitary gauge in order to interpret $Z_{2}$ monopoles in the D-brane picture. For simplicity we consider the case of $\nu_{2}=1$. Passing to the unitary gauge leaves a Dirac string with the pure gauge of the unbroken $U(2)$ subgroup around the string representing the non-trivial element of $\pi_{4}(U(2))$. Just as in Sec. 6, we can use the suspension map $\Sigma: \pi_{3}\left(U(2) / U(1)^{2}\right) \rightarrow \pi_{4}(U(2))$ to construct a field configuration carrying the monopole charge. Consider the volume of a 6-brane as the 'world-volume' of a 5-brane. The magnetic charges of 5-brane monopoles take values in $\pi_{3}(U(2))$ and, hence, have a colour $U(2)$ orientation. If we take a monopole and an anti-monopole created at some point on the 6-brane, separate them, adiabatically rotate the colour orientation of the monopole through $2 \pi$, and then annihilate the pair; the $U(2)$ pure gauge on the surface (homeomorphic to $S^{4}$ ) surrounding the trajectories of the Dirac strings of the monopole and the anti-monopole will be $U^{-1} \mathrm{e}^{2 \pi i \sigma^{3} \tau} U$, where $U$ is initial colour orientation of the monopole and $\tau$ is the 'time' direction on the 6-brane. This pure gauge represents a non-trivial element of $\pi_{4}(U(2))$ (see Sec. (6).

Since monopoles on 5-branes are represented by open D-strings, the monopole loop on the 6-brane, which carries $Z_{2}$ magnetic charge, corresponds to a cylindrical open D2 brane stretched between D6 branes with RR charges $N_{i}=2$ and $N_{j}>2$ (such a D6 brane exists, since $n_{3}>0$ ), see fig. 2. The $\mathrm{D} 2$ brane is protected from shrinking to a point by vacuum expectation values of massless modes of 2-6 fundamental strings. 


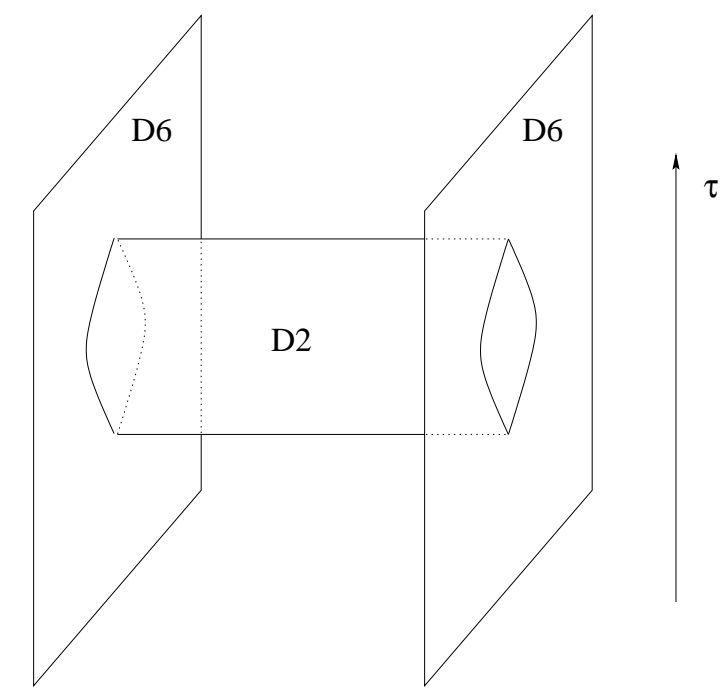

Figure 2: D-brane configuration describing $Z_{2}$ monopole on D6 brane.

\section{$7.2 \quad n_{3}=0$}

In this case $G=U\left(2 \nu_{2}+\nu_{1}\right)$ and $H=U(2)^{\nu_{2}} \times U(1)^{\nu_{1}}$, where $\nu_{1}$ is a number of charge 1 branes. We can assume that $\nu_{2}>0$, since the case of $U(N) / U(1)^{N}$ is considered above.

The first homomorphism in the exact sequence (7.1) is trivial now, since $n_{3}=0$, and we conclude that $\pi_{5}(G / H) / Z=\left(Z_{2}\right)^{\nu_{2}}$. This leaves two possibilities: $(1) \pi_{5}(G / H)=Z \otimes\left(Z_{2}\right)^{\nu_{2}}$ or $(2) \pi_{5}(G / H)=Z \otimes\left(Z_{2}\right)^{\nu_{2}-1}$. In a special case of $\nu_{1}=\nu_{2}=1$ the vacuum manifold is $U(3) / U(2) \times U(1)=\mathbf{C} \mathbf{P}^{2}$ and the latter possibility is realized: $\pi_{5}\left(\mathbf{C P}^{2}\right)=Z$ [24].. In general, we also have:

$$
\pi_{5}(G / H)=Z \otimes\left(Z_{2}\right)^{\nu_{2}-1} .
$$

The proof of this equality is sketched in Appendix B.

Part of the topological defects carrying charges of homotopy groups (7.2) are instantons and are described by embedded D0 branes and part of them are $Z_{2}$ monopoles described by open D2 branes stretched between adjacent charge 2 D6 branes.

There is, however, one subtlety. Consider, for brevity, the case of $\nu_{2}=1$, so that $G=U(N)$ and $H=U(2) \times U(1)^{N-2}$. The homotopy group $\pi_{5}\left(U(N) / U(2) \times U(1)^{N-2}\right)$ is $Z$, so apparently there are no monopoles. But, since $\pi_{5}\left(U(N) / U(2) \times U(1)^{N-2}\right) / \pi_{5}(U(N))=$ $\pi_{4}(U(2))=Z_{2}$, the group $\pi_{5}(U(N))$ forms $2 Z$ subgroup of $\pi_{5}\left(U(N) / U(2) \times U(1)^{N-2}\right)$. Therefore, only defects with even charge are related to large $U(N)$ gauge transformations at infinity and can be interpreted as instantons of the broken symmetry group. The instanton number, $k=\frac{1}{48 \pi^{3}} \int \operatorname{tr} F \wedge F \wedge F$, is equal to one half of the topological charge, if we normalise it in such a way that the generator of $\pi_{5}\left(U(N) / U(2) \times U(1)^{N-2}\right)$ has a unit charge. So, $k$ D0 branes embedded into D6 brane carry charge $2 k$. Odd-charged defects cannot be smoothly transformed into the unitary gauge. A Dirac string with $Z_{2}$ flux is always left. In a sense, odd-charged defects can be interpreted as fractionally charged instantons. 


\begin{tabular}{|c|c|c|c|}
\hline $\begin{array}{c}\text { Dp brane } \\
\text { configuration }\end{array}$ & Homotopy group & $\begin{array}{c}\text { Type of } \\
\text { topological defects }\end{array}$ & D-brane description \\
\hline $\mathrm{p}=2$ & $\pi_{1}(U(N))=Z$ & $U(1)$ vortices & D0 branes \\
\hline$\overline{p=3}$ & $\bar{\pi}_{2}(G / H)=Z^{m-1}$ & monopoles & open D1 branes \\
\hline $\begin{array}{l}\mathbf{p}=\mathbf{4} \\
N \geq 2\end{array}$ & $\begin{array}{c}\pi_{3}(U(N))=Z \\
\pi_{3}\left(U(N) / U(1)^{N}\right)=Z\end{array}$ & instantons & D0 branes \\
\hline $\begin{array}{l}\mathbf{p}=\mathbf{5} \\
N>2 \\
n_{2}>0\end{array}$ & $\pi_{4}(G / H)=Z^{n_{2}-1}$ & monopoles & open D1 branes \\
\hline $\begin{array}{l}\mathbf{p}=\mathbf{5} \\
N=2\end{array}$ & $\begin{array}{c}\pi_{4}(U(2))=Z_{2} \\
\pi_{4}\left(U(2) / U(1)^{2}\right)=Z_{2}\end{array}$ & $Z_{2}$ instantons & closed D1 branes \\
\hline $\begin{array}{l}\mathbf{p}=\mathbf{6} \\
N \geq 3\end{array}$ & $\begin{array}{c}\pi_{5}(U(N))=Z \\
\pi_{5}\left(U(N) / U(1)^{N}\right)=Z\end{array}$ & instantons & D0 branes \\
\hline $\begin{array}{l}\mathbf{p}=\mathbf{6} \\
N=2\end{array}$ & $\begin{array}{c}\pi_{5}(U(2))=Z_{2} \\
\pi_{5}\left(U(2) / U(1)^{2}\right)=Z_{2}\end{array}$ & $Z_{2}$ instantons & $?$ \\
\hline $\begin{array}{l}\mathbf{p}=\mathbf{6} \\
n_{3}>0\end{array}$ & $\pi_{5}(G / H)=\left(Z_{2}\right)^{\nu_{2}}$ & $Z_{2}$ monopoles & cylindrical D2 branes \\
\hline \multirow{3}{*}{$\begin{array}{c}\mathbf{p}=\mathbf{6} \\
n_{3}=0 \\
\nu_{2}>0\end{array}$} & \multirow{3}{*}{$\pi_{5}(G / H)=Z \otimes\left(Z_{2}\right)^{\nu_{2}-1}$} & $Z_{2}$ monopoles & cylindrical D2 branes \\
\hline & & $\begin{array}{c}\text { instantons } \\
(Z \text { charge even }) \\
\end{array}$ & D0 branes \\
\hline & & $\begin{array}{c}Z_{2} \text { monopoles } \\
(Z \text { charge odd })\end{array}$ & $?$ \\
\hline
\end{tabular}

Table 1: Topological classification of soliton states on D-branes; $n_{k}$ is a number of Dp branes with RR charge $N_{i} \geq k, \nu_{k}$ is a number of Dp branes with RR charge $N_{i}=k$.

\section{Discussion.}

We have presented a complete classification of topological charges on stable BPS D-branes of type II string theories. In almost all cases it is possible to construct D-brane configuration representing a state with a given topological charge. The results are summarised in Table 1.

Generically, embedded topological defects are described either by D0 branes in type IIA theory or by open D1 branes in type IIB theory and there are BPS states in any charged sector preserving some part of the super-symmetry.

The exceptions to this rule are $Z_{2}$ instantons on D5 branes and $Z_{2}$ monopoles on D6 branes, which are described by closed D1 branes and by cylindrical open D2 branes, respectively. These branes are necessarily curved and therefore cannot preserve the supersymmetry. The non-trivial topology of these brane configurations resides in the massless modes of the fundamental strings stretched between the embedded and embracing branes.

We also find topological defects on certain configurations of D6 branes which can be interpreted as fractionally charged instantons and, on the other hand, share common features with $Z_{2}$ monopoles. We have not been able to find a D-brane interpretation of these objects. 


\section{Acknowledgements}

We are grateful to J. Abmjørn for hospitality at The Niels Bohr Institute where this work has been completed. We also thank Sandy Rutherford for a helpful discussion.

\section{Appendix A Stable homotopy groups of flag mani- folds}

In this Appendix, we shall sketch the calculation of the homotopy groups $\pi_{n}(G / H), G=$ $U(N), H=U\left(N_{1}\right) \times \ldots \times U\left(N_{m}\right)$ for $n \leq 5, N_{i}>2$. These groups can be calculated from the exact sequence of the fibre bundle

$$
G \stackrel{H}{\rightarrow} G / H
$$

For odd $n=2 k+1$ we consider the exact sequence

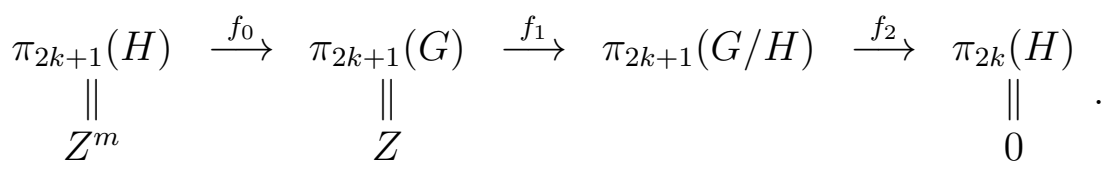

First, we note that the image of any of the $m$ generators of $\pi_{2 k+1}(H)$ under the homomorphism $f_{0}$ is the canonical generator of $\pi_{2 k+1}(G)$. Indeed, the upper left corner immersion $U\left(N_{1}\right) \rightarrow U(N)$ induces the isomorphism of the homotopy groups

$$
\pi_{2 k+1}\left(U\left(N_{1}\right)\right)=\pi_{2 k+1}(U(N))
$$

for sufficiently large $N_{1}, N$. The above statement means that the image of the mapping $f_{0}$ is the whole group $\pi_{2 k+1}(G)$. The exactness of the sequence, i.e. that $\operatorname{Im} f_{0}=\operatorname{Ker} f_{1}$, then implies that $f_{1}$ is a trivial mapping, $\operatorname{Im} f_{1}=0$. $\operatorname{In} \operatorname{turn}, \operatorname{Im} f_{1}=\operatorname{Ker} f_{2}$, so $\operatorname{Ker} f_{2}=0$ and $f_{2}$ is an isomorphism. This is only possible if

$$
\pi_{2 k+1}(G / H)=0 .
$$

which is our first result.

In the case of even $n=2 k$ we consider the long exact sequence

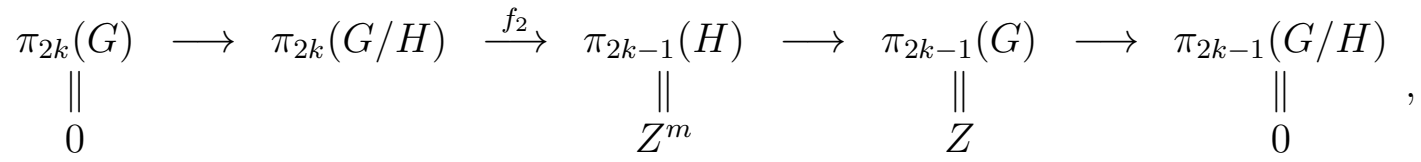

which implies that

$$
Z^{m} / \pi_{2 k}(G / H)=Z \quad
$$

Consequently,

$$
\pi_{2 k}(G / H)=Z^{m-1} .
$$




\section{Appendix B Homotopy groups $\pi_{5}\left(U\left(2 \nu_{2}+\nu_{1}\right) / U(2)^{\nu_{2}} \times\right.$$$
\left.U(1)^{\nu_{1}}\right)
$$

Consider the fibre bundle

$$
U(N) / U(2)^{\nu_{2}} \stackrel{U(1)^{\nu_{1}}}{\longrightarrow} U(N) / U(2)^{\nu_{2}} \times U(1)^{\nu_{1}}
$$

and the short exact sequence

$$
\begin{gathered}
\pi_{5}\left(U(1)^{\nu_{1}}\right) \longrightarrow \pi_{5}\left(U(N) / U(2)^{\nu_{2}}\right) \longrightarrow \pi_{5}\left(U(N) / U(2)^{\nu_{2}} \times U(1)^{\nu_{1}}\right) \\
\quad \| \\
0
\end{gathered}
$$

from which we infer that

$$
\pi_{5}\left(U(N) / U(2)^{\nu_{2}} \times U(1)^{\nu_{1}}\right)=\pi_{5}\left(U(N) / U(2)^{\nu_{2}}\right)
$$

and we can study the latter homotopy groups instead.

It is instructive to consider separately the case of $\nu_{2}=1$. From the exact sequence (7.1) we know that

$$
\pi_{5}(U(N) / U(2)) / \pi_{5}(U(N))=\pi_{4}(U(2))
$$

and that we have to distinguish between two possibilities:

(1) $\pi_{5}(U(N) / U(2))=\pi_{5}(U(N)) \otimes \pi_{4}(U(2))=Z \otimes Z_{2}$

or

(2) $\pi_{5}(U(N) / U(2))=Z$.

If $N=2$, the homotopy group of interest is $Z$, as $U(3) / U(2)=S^{5}$, which means that $\pi_{5}(U(3))$ is the $2 Z$ subgroup of $\pi_{5}(U(3) / U(2))$. Suppose that $\pi_{5}(U(N) / U(2))=Z \otimes Z_{2}$ for some $N$, then $\pi_{5}(U(N))$ is the $Z \otimes\{1\}$ subgroup of $\pi_{5}(U(N) / U(2))$. The immersion of $U(3) / U(2)$ into $U(N) / U(2)$ induces a homomorphism of homotopy groups

$$
f: \pi_{5}(U(3) / U(2)) \rightarrow \pi_{5}(U(N) / U(2)) .
$$

Since any map from $S^{5}$ to $U(N)$ can be reduced to the $U(3)$ subgroup, we have: $f(2)=$ $f(1,0)$, which leaves no room for $f(1)$ in $\pi_{5}(U(N) / U(2))$. Thus we conclude that

$$
\pi_{5}(U(N) / U(2))=Z
$$

for any $N$.

Now, consider the fibre bundle

$$
U(N) / U(2) \stackrel{U(2)^{\nu_{2}-1}}{\longrightarrow} U(N) / U(2)^{\nu_{2}}
$$

Analysis of the exact sequence

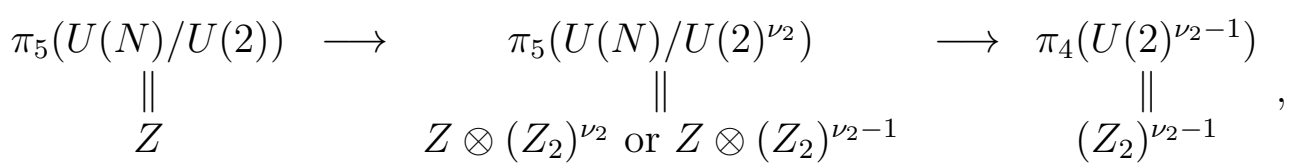

demonstrates the validity of eq. (7.2). 


\section{References}

[1] E. Witten, Nucl. Phys. B460 (1996) 335, hep-th/9510135.

[2] A. Giveon and D. Kutasov, hep-th/9802067.

[3] R. Minasian and G. Moore, JHEP 9711 (1997) 002, hep-th/9710230.

[4] E. Witten, JHEP 9812 (1998) 019, hep-th/9810188.

[5] H. Garcia-Compean, hep-th/9812226.

[6] S. Gukov, hep-th/9901042.

[7] E.R. Sharpe, hep-th/9902116.

[8] A. Sen, JHEP 9809 (1998) 023, hep-th/9808141; 9812 (1998) 021, hep-th/9812031; hepth/9902105.

[9] P. Hořava, hep-th/9812135.

[10] P. Yi, hep-th/9901159.

[11] O. Bergman, E.G. Gimon and P. Hořava, hep-th/9902160.

[12] H. Awata, S. Hirano and Y. Hyakutake, hep-th/9902158.

[13] M.R. Douglas, hep-th/9512077.

[14] M. Green, J.A. Harvey and G. Moore, Class. Quant. Grav. 14 (1997) 47, hepth/9605033.

[15] Y.-K.E. Cheung and Z. Yin, Nucl. Phys. B517 (1998) 69, hep-th/9710206.

[16] A. Strominger, Phys. Lett. B383 (1996) 44, hep-th/9512059.

[17] D.-E. Diaconescu, Nucl. Phys. B503 (1997) 220, hep-th/9608163.

[18] C. Vafa, Nucl. Phys. B463 (1996) 435, hep-th/9512078.

[19] M.R. Douglas, hep-th/9604198.

[20] J.L.F. Barbón and A. Pasquinucci, Nucl. Phys. B517 (1998) 125, hep-th/9708041.

[21] W. Taylor, hep-th/9801182.

[22] A.S. Schwarz, Quantum Field Theory and Topology (Springer-Verlag, 1993).

[23] Y.M. Cho, Phys. Lett. B81 (1979) 25.

[24] B.A. Dubrovin, A.T. Fomenko and S. P. Novikov, Modern Geometry - Methods and Applications, p. II (Springer, 1990).

[25] W. Taylor, Nucl. Phys. B508 (1997) 122, hep-th/9705116.

[26] R. Argurio, F. Englert, L. Houart and P. Windey, Phys. Lett. B408 (1997) 151, hepth/9704190.

[27] J. Polchinski, hep-th/9611050.

[28] P.K. Townsend, Phys. Lett. B373 (1996) 68, hep-th/9512062.

[29] O.J. Ganor, S. Ramgoolam and W. Taylor, Nucl. Phys. B492 (1997) 191, hepth/9611202.

[30] A.A. Tseytlin, Nucl. Phys. B501 (1997) 41, hep-th/9701125. 
[31] C. Bachas, hep-th/9806199.

[32] H. Liu and A.A. Tseytlin, hep-th/9903091.

[33] J.H. Schwarz, Nucl. Phys. Proc. Suppl. 55B (1997) 1, hep-th/9607201.

[34] O. Bergman, Nucl. Phys. B525 (1998) 104, hep-th/9712211.

[35] K. Hashimoto, H. Hata and N. Sasakura, Phys. Lett. B431 (1998) 303, hep-th/9803127.

[36] T. Kawano and K. Okuyama, Phys. Lett. B432 (1998) 338, hep-th/9804139.

[37] O. Bergman and B. Kol, Nucl. Phys. B536 (1998) 149, hep-th/9804160.

[38] D. Bak, K. Hashimoto, B.-H. Lee, H. Min and N. Sasakura, hep-th/9901107.

[39] T. Banks and M.B. Green, JHEP 05 (1998) 002, hep-th/9804170.

[40] C.-S. Chu, P.-M. Ho and Y.-Y. Wu, Nucl. Phys. B541 (1999) 179, hep-th/9806103.

[41] I.I. Kogan and G. Luzón, Nucl. Phys. B539 (1999) 121, hep-th/9806197.

[42] C. Park and S.-J. Sin, hep-th/9807156.

[43] M. Bianchi, M.B. Green, S. Kovacs and G. Rossi, JHEP 9808 (1998) 013, hepth/9807033.

[44] N. Dorey, V.V. Khoze, M.P. Mattis and S. Vandoren, Phys. Lett. B442 (1998) 145, hep-th/9808157.

[45] A. Bilal and C.-S. Chu, hep-th/9810195.

[46] E.T. Akhmedov, hep-th/9812038.

[47] M.R. Douglas, S. Katz and C. Vafa, Nucl. Phys. B497 (1997) 155, hep-th/9609071.

[48] W. Nahm, Phys. Lett. B96 (1980) 323.

[49] A.T. Fomenko, D.B. Fuchs and V.L. Gutenmacher, Homotopic topology, (Akadémiai Kiadó, Budapest, 1986). 